\title{
Alteraciones de la tolerancia a la glucosa y frecuencia de síndrome metabólico en pacientes con enfermedad por hígado graso no alcohólico
}

A. Verónica Araya $Q^{1}$, José $M$ Valera $M^{2}$, Jorge Contreras $B^{2}$, Attila Csendes $J^{3}$, Juan C Díaz J ${ }^{3}$, Patricio Burdiles $\mathrm{P}^{3}$, Jorge Rojas $\mathrm{C}^{3}$, Fernando Maluenda $\mathrm{G}^{3}$, G ladys Smok $\mathrm{S}^{4}$, Jaime Poniachik $\mathrm{T}^{2}$.

Glucose tolerance alterations and frequency of metabolic syndrome among patients with non alcoholic fatty liver disease

Background: Non alcoholic fatty liver disease (NAFLD) is associated to diabetes mellitus, obesity, disturbances in serum lipid levels, insulin resistance and metabolic syndrome. Aim: To assess glucose tolerance and the presence of metabolic syndrome among patients with biopsy proven NAFLD. Patients and methods: Serum lipid levels, hepatic function tests were measured and an oral glucose tolerance test was performed in 46 patients (mean age $45 \pm 12$ years, 36 females) without history of diabetes mellitus and with steatosis in a liver biopsy. Results: Mean body mass index of the sample was $37 \pm 12 \mathrm{~kg} / \mathrm{m}^{2}$. Seventeen percent had pure steatosis, $78 \%$ had steatohepatitis with or without fibrosis and $50 \%$ had fibrosis in the liver biopsy. Glucose intolerance and diabetes was found in $57 \%$ and $15 \%$ of cases, respectively. The presence of steatohepatitis was higher in diabetics, compared with subjects with glucose intolerance or a normal glucose response (43, 38 and $8 \%$, respectively, $\mathrm{p}<0.0001$ ). Ninety three percent had a metabolic syndrome and the proportion of biopsies with fibrosis was higher among subjects with more than three diagnostic criteria for metabolic syndrome compared with those with three or less criteria (59 and $46 \%$, respectively, $p$ $\varangle$ 0.05). Conclusions: Glucose intolerance, diabetes and metabolic syndrome are common among patients with NAFLD, even when they are not obese (Rev Méd Chile 2006; 134: 1092-8).

(Key words: Fatty liver, non-alcoholic; Glucose tolerance test; Insulin resistance; Metabolic syndrome)

Recibido el 6 de enero, 2006. Aceptado el 30 de marzo, 2006.

${ }^{1}$ Sección de Endocrinología, ${ }^{2}$ Centro de Gastroenterología, Departamento de Medicina,

${ }^{3}$ Departamento de Cirugía, ${ }^{4}$ Departamento de Anatomía Patológica, Hospital Clínico de la Universidad de Chile.

Correspondencia a: Dr. Jaime Poniachik. Centro de Gastroenterología, Hospital Clínico Universidad de Chile. Santos Dumont 999, Santiago-Chile. Fax: (56-2) 9788353.

E-mail: jponiach@redclinicauchile.cl

Dra. Verónica Araya. Sección Endocrinología. Hospital Clínico U. de Chile. Santos

Dumont 999. Santiago de Chile. Fax: (56-2) 777 6891. E-mail: varaya@redclinicauchile.cl 
L a enfermedad por hígado graso no alcohólico (EHGNA) es una entidad clínico-patológica reconocida en las últimas décadas. Se define como la presencia de esteatosis hepática en más de $5 \%$ de los hepatocitos, con o sin la presencia de inflamación y fibrosis, en un paciente con una ingesta alcohólica menor de $40 \mathrm{~g}$ a la semana e idealmente, sin otras causas de daño hepático crónico. La prevalencia, según algunas series extranjeras, es de alrededor de $20 \%$ en la población general ${ }^{1-3}$. La histología puede progresar desde esteatosis pura a esteatohepatitis, fibrosis e incluso cirrosis, al igual que en la enfermedad hepática alcohólica. Se ha descrito que 20 a 30\% puede llegar a cirrosis después de 20 años y potencialmente llevar a falla hepática y hepatocarcinoma. La presentación clínica y de laboratorio también es similar a la enfermedad hepática por alcohol, salvo en algunas ocasiones, en que la alanino aminotransferasa (GPT) es mayor que la aspartato aminotransferasa (GOT). Excepcionalmente puede producirse un cuadro similar a la hepatitis alcohólica.

La EHGNA se asocia a obesidad, diabetes mellitus y dislipidemia ${ }^{3-6}$. En un estudio publicado por nuestro grupo, el análisis de la biopsia hepática de obesos mórbidos demostró $91 \%$ de esteatosis, $47 \%$ de fibrosis, $45 \%$ de esteatohepatitis y $1,4 \%$ de cirrosis $^{7}$.

En el último tiempo, se ha reconocido la asociación de insulino resistencia (IR) e hígado graso. Diversos estudios han demostrado que los pacientes con EHGNA presentan hiperinsulinemia, disminución del índice de sensibilidad insulínica y presencia de elementos del síndrome metabólico como obesidad abdominal, alteraciones de la tolerancia a la glucosa, hipertrigliceridemia e hipertensión arterial ${ }^{6,8,9}$. La IR se encontraría presente aun en los pacientes no obesos y el riesgo de esteatosis aumenta cuando se asocia a un mayor número de componentes del síndrome metabólico ${ }^{9-12}$. Algunos estudios han demostrado una alta prevalencia de intolerancia a glucosa y diabetes en pacientes con EHGNA ${ }^{6,11}$ y su presencia aumentaría el riesgo de progresión a fibrosis 9 .

El objetivo del presente trabajo fue evaluar en pacientes con alteraciones histológicas compatibles con EHGNA, demostrada en biopsia hepática, la presencia de alteraciones de la tolerancia a la glucosa y de elementos del síndrome metabólico.

\section{PACIENTES Y MÉTODO}

Se seleccionaron las biopsias hepáticas realizadas en el Hospital Clínico de la Universidad de Chile, entre los años 1999 y 2003, en las cuales se encontró algún grado de esteatosis. Se contactó a los pacientes que no tenían el diagnóstico conocido de intolerancia a glucosa o diabetes o ingesta alcohólica superior a $40 \mathrm{~g}$ a la semana y que tuvieran antígeno de superficie para hepatitis B y anticuerpo antivirus $C$ negativos. Se excluyó a los pacientes que se encontraran recibiendo fármacos reconocidos como potencialmente hepatotóxicos o corticoides. Todos los pacientes tenían anticuerpos antinucleares, antimitocondriales y antimúsculo liso negativos, estudio de cinética de fiemo, feritina y cenuloplasmina normales. También se confirmó que los niveles de tiroxina libre y TSH se encontraran dentro del rango normal.

A todos ellos se les realizó anamnesis y examen físico, consignando presión arterial, peso y talla para cálculo del índice de masa corporal (IMC) y perímetro de cintura.

Después de un ayuno de $10 \mathrm{~h}$, se les realizó una prueba de tolerancia oral con $75 \mathrm{~g}$ de glucosa, tomando muestras cada 30 min durante $2 \mathrm{~h}$, para la medición de glicemia. Esta determinación se realizó por el método de glucosa oxidasa. Además, se contó con la determinación de perfil lipídico y hepático.

Las alteraciones de tolerancia a la glucosa se clasificaron de acuerdo a los criterios actuales definidos por la Asociación Americana de Diabetes (ADA). Según glicemia de ayunas: intolerancia a la glucosa de ayuno con glicemia entre 100 y 125 mg/ dl y diabetes con glicemia $\geq 126 \mathrm{mg} / \mathrm{dl}$; según la glicemia a las dos horas de la sobrecarga de glucosa: intolerancia a glucosa con glicemia entre 141 y 199 $\mathrm{mg} / \mathrm{dl}$ y diabetes con glicemia $\geq 200 \mathrm{mg} / \mathrm{dl}^{13}$.

Para definir la presencia de síndrome metabólico se utilizaron los criterios clínicos recientemente propuestos por la International Diabetes Federation (IDF): obesidad central definida por un perímetro de cintura $\geq 90 \mathrm{~cm}$ en el hombre y $\geq 80$ $\mathrm{cm}$ en la mujer y al menos dos de los siguientes criterios: colesterol HDL $<40 \mathrm{mg} / \mathrm{dl}$ en el hombre y $<50 \mathrm{mg} / \mathrm{dl}$ en la mujer; triglicéridos $\geq 150 \mathrm{mg} / \mathrm{dl}$; glicemia de ayuno $\geq 100 \mathrm{mg} / \mathrm{dl}$ y presión arterial $\geq 130 / \geq 85 \mathrm{mmHg}$. La presencia de 3 o más criterios definen la presencia de síndrome metabólico ${ }^{14}$.

Estadística. Para la comparación entre grupos se aplicaron los tests de $\chi^{2}$ y Kruskal-Wallis y para 
evaluar la relación entre variables se utilizó el coeficiente de correlación de Spearman-rho. Para el cálculo del área bajo la curva de glicemia $\left(\mathrm{ABC}_{\mathrm{GLICEMA}}\right)$, se utilizó el cálculo de la integral bajo el área de la curva concentración-tiempo. Los resultados se expresan como promedio \pm DS.

Este protocolo fue aprobado por el Comité de Etica del Hospital Clínico de la Universidad de Chile $\mathrm{y}$ todos los pacientes firmaron un consentimiento informado antes de ser sometidos al estudio.

\section{Resultados}

El grupo estudiado incluyó 46 pacientes, 36 mujeres y 10 hombres, con un rango de edad entre 19 y 62 años. Las características de los pacientes se presentan en la Tabla 1.

En la biopsia hepática se observó esteatosis pura en $17,4 \%$, esteatohepatitis con o sin fibrosis en $78,3 \%$ y $50 \%$ del grupo total presentaba fibrosis. De acuerdo a la definición usada previamente por nuestro grupo ${ }^{7}$, la esteatosis fue leve (presente en menos de $25 \%$ de los hepatocitos) en $39,2 \%$ (18/46), moderada (25 a 50\% de los hepatocitos) en $30,4 \%(14 / 46)$ y severa $(>50 \%$ de los hepatocitos) en 30,4\% (14/46).
La GOT estuvo elevada en $48,9 \%$ de los casos $(63,7 \pm 110 \mathrm{UI} / \mathrm{l})$, la GPT en $69,8 \%(73,5 \pm 50,6 \mathrm{UI} / \mathrm{l})$ y la gamma glutamil transpeptidasa (GGT) en $37,8 \%(100 \pm 161,9 \mathrm{UI} / \mathrm{l})$.

Del total de pacientes, 44 tuvo el IMC mayor a 26 $\mathrm{kg} / \mathrm{m}^{2}, 63 \%$ tenía IMC entre 26 y 39 y 32,6\% IMC $>40$ $\mathrm{kg} / \mathrm{m}^{2}$. De acuerdo al perímetro de cintura 44 tenían obesidad central ya que sólo dos mujeres tuvieron un perímetro de cintura menor a $80 \mathrm{~cm}$. Nueve pacientes tenían hipertensión arterial diagnosticada previamente y uno de ellos se encontraba sin tratamiento.

El $66,7 \%$ de los casos tuvo colesterol LDL mayor de $100 \mathrm{mg} / \mathrm{dl}$ y $57 \%$ tenía triglicéridos sobre 150 $\mathrm{mg} / \mathrm{dl}$. El colesterol HDL estaba bajo $50 \mathrm{mg} / \mathrm{dl}$ en 21 mujeres y bajo $40 \mathrm{mg} / \mathrm{dl}$ en 4 hombres.

De los 46 pacientes, 47,8\% tenía glicemia de ayuno entre 100 y $125 \mathrm{mg} / \mathrm{dl}$ y 4,3\% una glicemia $\geq 126 \mathrm{mg} / \mathrm{dl}$. Analizando el valor de la glicemia a las dos horas de la sobrecarga de glucosa, 56,5\% fue intolerante a la glucosa y 15,2\% cumplió el criterio de diabetes (Figura 1).

La proporción de casos con esteatohepatitis fue significativamente mayor en el grupo con diabetes e intolerancia a la glucosa, comparado con los normoglicémicos, según el valor de glicemia a las $2 \mathrm{~h}$ post sobrecarga ( $43 \%$ vs $38 \%$ vs $8 \%$, respectivamente, p $\varangle 0,0001$ ), pero no hubo diferencia significativa entre

Tabla 1. C aracterísticas clínicas y de laboratorio de 46 pacientes con el diagnóstico histológico de EH G N A

\begin{tabular}{|c|c|c|}
\hline & Promedio $\pm \mathrm{DE}$ & Rango normal \\
\hline Edad (años) & $44,9 \pm 12,1(19-62)$ & \\
\hline IMC $\left(\mathrm{kg} / \mathrm{m}^{2}\right)$ & $37 \pm 11,9(25,2-72,4)$ & \\
\hline Perímetro de cintura (cm) & $106,3 \pm 22,5(78-176)$ & \\
\hline GOT (UI/l) & $63,7 \pm 110(13-760)$ & $14-36$ \\
\hline GPT (UI/l) & $73,5 \pm 50,6(17-253)$ & $9-52$ \\
\hline GGT (UI/l) & $100 \pm 161,9(17-989)$ & $8-78$ \\
\hline Fosfatasas alcalinas (UI/l) & $111,9 \pm 81,6(45-572)$ & $38-126$ \\
\hline Protrombina (\%) & $95,4 \pm 8,6(67-100)$ & $70-100$ \\
\hline Albumina $(\mathrm{g} / \mathrm{dl})$ & $4 \pm 0,4(2,9-4,8)$ & $3,5-5$ \\
\hline Colesterol total (mg/dl) & $210 \pm 44(154-307)$ & $130-200$ \\
\hline Colesterol HDL (mg/dl) & $44,1 \pm 9,3(23-66)$ & $35-55$ \\
\hline Colesterol LDL (mg/dl) & $129,6 \pm 36,8(64-209)$ & $0-140$ \\
\hline Triglicéridos (mg/dl) & $169 \pm 88,3(82-541)$ & $35-150$ \\
\hline Glicemia basal (mg/dl) & $102,2 \pm 14,2(83-152)$ & $60-99$ \\
\hline Glicemia $120 \mathrm{~min}(\mathrm{mg} / \mathrm{dl})$ & $160,4 \pm 43,6(107-284)$ & $<140$ \\
\hline $\mathrm{ABC}_{\mathrm{GLUCOSA}}(\mathrm{mg} / \mathrm{ml} / \mathrm{min})$ & $19320,1 \pm 3814,5(13.410-28.410)$ & \\
\hline
\end{tabular}

GOT: aminotransferasa glutámico oxalo-acética; GPT: alanina aminotransferasa pirúvica; GGT: gamma glutamil transpeptidasa. 


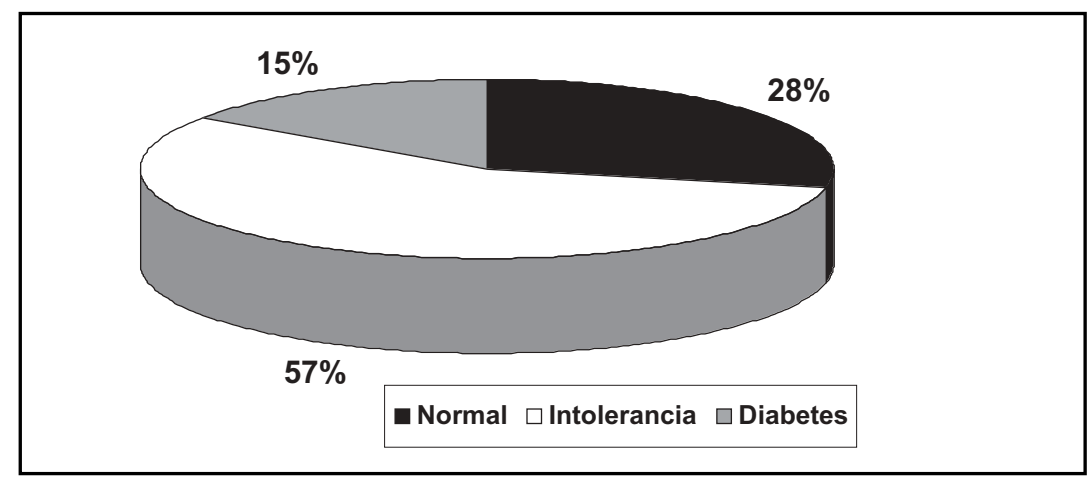

Figura 1. Frecuencia de alteraciones de la glicemia a las $2 \mathrm{~h}$ poscarga con $75 \mathrm{~g}$ de glucosa en 46 pacientes con diagnóstico histológico de EHGNA.

diabéticos e intolerantes a la glucosa (Figura 2). La proporción de casos con fibrosis fue significativamente mayor en el grupo con diabetes comparado con los intolerantes (57\% vs $42 \%$, p $\varangle$ 0,05). Al evaluar según la severidad de cada tipo de lesión histológica, no hubo diferencias significativas entre pacientes con normoglicemia, intolerancia a la glucosa o diabetes.

Al separar a los pacientes de acuerdo al tipo histológico, esteatosis pura, esteatohepatitis sin fibrosis y fibrosis, el IMC fue significativamente mayor en el grupo con esteatosis pura comparado con el grupo con fibrosis y esteatohepatitis ( $p<0,005$ ). No hubo diferencia significativa para el perímetro de cintura. Tampoco para el valor de glicemia basal y a los 120 min post sobrecarga, el $\mathrm{ABC}_{\mathrm{GLCEMA}}$, colesterol total, LDL, HDL y triglicéridos.
La GPT estuvo elevada en una mayor proporción de casos con esteatohepatitis (con o sin fibrosis) comparado con el grupo con esteatosis pura, pero esta diferencia no fue significativa ( $p=0,07$ ). La GOT y la GPT se correlacionaron positivamente con el grado de inflamación lobulillar ( $r=0,51, p<0,0005$ y $r=0,38, p \varangle 0,005)$. La GOT además se correlacionó con el grado de fibrosis ( $r=0,39, p<0,05$ ).

$\mathrm{Al}$ agrupar a los pacientes de acuerdo al IMC, la proporción de casos con esteatohepatitis $\mathrm{y}$ fibrosis fue significativamente mayor en los con IMC entre 26 y $40 \mathrm{~kg} / \mathrm{m}^{2}$, comparados con los con IMC $>40$ (35\% vs $20 \%$ p $=0,01$ y $58,6 \%$ vs $40 \%$ p $=0,005$ respectivamente). La severidad de la inflamación lobulillar fue significativamente mayor en el grupo con IMC $<30$ ( $p<0,005)$.

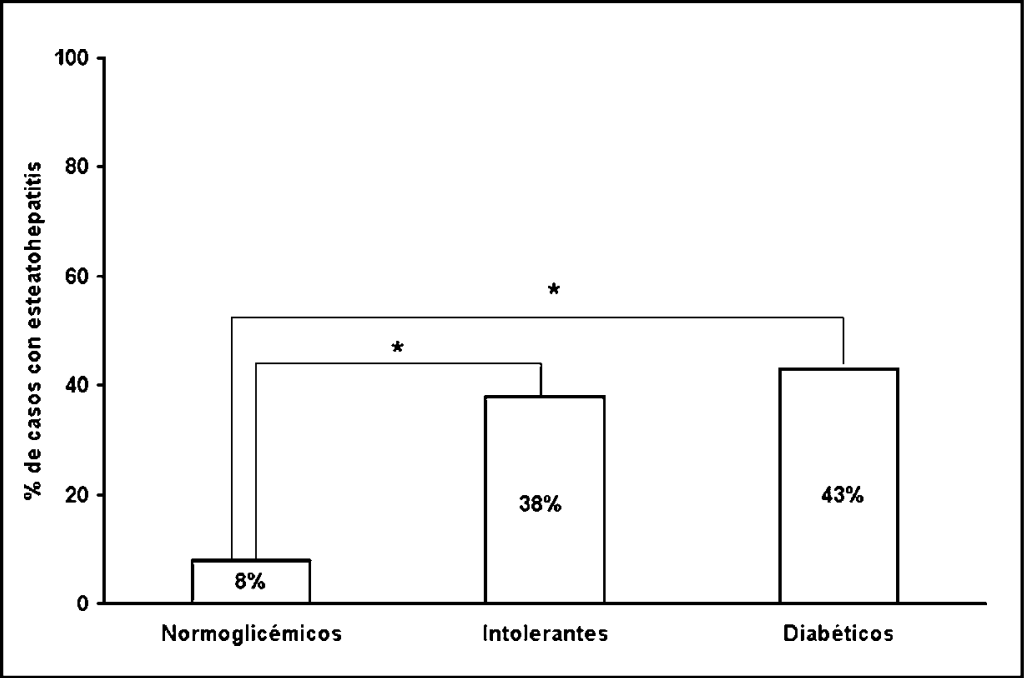

Figura 2. Proporción de casos con esteatohepatitis según alteración de la tolerancia a la glucosa a las 2 h poscarga. $* p=0,0001$. 
De acuerdo a parámetros del perfil lipídico, sólo encontramos una proporción significativamente mayor de casos con esteatohepatitis en el grupo con triglicéridos $>150 \mathrm{mg} / \mathrm{dl}(\mathrm{p}=0,0001)$ y de fibrosis en el grupo con HDL $<40 \mathrm{mg} / \mathrm{dl}$ ( $\mathrm{p}<0,005)$. El nivel de colesterol HDL se correlacionó inversamente con el grado de esteatosis $(r=0,37 \mathrm{p}=0,02)$.

De acuendo a los criterios clínicos actuales, propuestos por la IDF, 43 pacientes $(93,5 \%)$ presentaron un síndrome metabólico. Diecisiete de ellos tenían más de 3 criterios y la proporción de biopsias con fibrosis fue significativamente mayor en este grupo, comparados con los que tenían sólo 3 criterios ( $59 \%$ vs $46 \%, p<0,05$ ).

\section{Discusión}

Nuestros resultados muestran que más de $70 \%$ de los pacientes con EHGNA presenta alteraciones de la tolerancia a la glucosa, incluso en sujetos con glicemia de ayuno normal y sin obesidad. La frecuencia de diabetes tipo 2 alcanza hasta $50 \%$ en estos casos ${ }^{10,15}$. En nuestro grupo encontramos $15 \%$ de diabetes, siendo más frecuente la intolerancia a la glucosa, lo que concuerda con lo publicado por otros autores ${ }^{6,16}$. Encontramos una proporción significativamente mayor de casos con esteatohepatitis en los intolerantes a la glucosa y diabéticos con respecto a los normales y la proporción de biopsias con fibrosis fue mayor en el grupo con diabetes. Esto concuerda con lo descrito, en que la intolerancia a la glucosa y la diabetes senían factores de progresión hacia fibrosis ${ }^{17}$.

$\mathrm{Al}$ igual que otros autores, observamos que los pacientes con triglicéridos sobre $150 \mathrm{mg} / \mathrm{dl}$ y con HDL bajo $40 \mathrm{mg} / \mathrm{dl}$ presentaron significativamente más esteatohepatitis y fibrosis, respectivamente ${ }^{15,18}$.

Casi todos nuestros casos tenían IMC mayor a 26 y $33 \%$ tenía obesidad mórbida. Se ha descrito que la obesidad severa se asocia a mayor grado de esteatosis y es un factor de riesgo para la progresión a esteatohepatitis y fibrosis ${ }^{7,16}$. Sin embargo, en este estudio observamos que, si bien los pacientes con esteatosis pura son los que presentan un IMC mayor, encontramos una proporción de esteatohepatitis y fibrosis significativamente mayor en el grupo con IMC $<40$ y la inflamación es más severa en los pacientes con IMC menor a 30. Esto, probablemente, se debe a que en algunos casos el compromiso hepático puede ser independiente del grado de obesidad y senian otros factores relacionados con la IR, los que favorecenían la progresión del daño. Otra observación fue que las alteraciones de la tolerancia a la glucosa fueron más frecuentes en los pacientes con IMC menores, incluso estaban presentes en los dos casos con IMC normal. Estos hallazgos resaltan la importancia de evaluar la tolerancia a la glucosa en todo paciente con EHGNA, aunque tenga peso normal.

Hace más de una década se describió la asociación de cirrosis hepática con hiperinsulinemia e $\mathrm{IR}^{19}$. Posteriormente se observó que este fenómeno se presenta hasta en $85 \%$ de los casos con EHGNA sin cirrosis ${ }^{6}$. Marchesini encontró, en pacientes con EHGNA y sin diabetes, un síndrome de IR en una proporción significativamente mayor de sujetos con esteatohepatitis, comparados con los con esteatosis pura ${ }^{17}$.

Recientemente, Ryan encontró síndrome metabólico en 65\% de pacientes con EHGNA y observó que estos pacientes presentaron un porcentaje mayor de fibrosis que los sin síndrome metabóli$\mathrm{co}^{11}$. En nuestro estudio, más de $90 \%$ de los pacientes con EHGNA presentaron criterios diagnósticos de síndrome metabólico, de acuerdo a la definición actual de la IDF y los casos que tenían más de tres criterios, presentaron fibrosis con mayor frecuencia, lo que concuerda con lo descrito por Ryan. Es por esto que algunos autores han planteado que la EHGNA sería la manifestación hepática del síndrome metabólico y que podría preceder en años a la aparición de la diabetes ${ }^{6,20}$.

Se ha postulado que la acción de la insulina sobre la expresión de factores de crecimiento, promovenáa la formación de matriz extracelular y determinana la progresión hacia fibrosis hepática ${ }^{21}$. Sin embargo, otros factores relacionados con IR pueden estar involucrados. El aumento del flujo de ácidos grasos desde el tejido adiposo intraabdominal hacia el hígado y el aumento de la peroxidación mitocondrial, estanían relacionados con el aumento de la resistencia a la acción de insulina y con la esteatosis y progresión a cimosis ${ }^{22-24}$. La liberación de citoquinas y el estrés oxidativo (EO) también participarían en la naturaleza multifactorial de esta enfermedad ${ }^{25}$.

Nuestros estudios han mostrado un aumento del EO en el hígado de pacientes con EHGNA con esteatosis y que esta condición está exacerbada en la esteatohepatitis. Esto llevaría a modular la función de la célula de Küpffer a través de la activación de factores de transcripción que aumentan la expresión 
de genes relacionados con mediadores proinflamatorios como el TNF- $\alpha$ o fibrogénicos, como el factor de crecimiento transformante-ß1 (TGF- $\beta 1)^{26}$.

El EO puede determinar depleción de ácidos grasos poliinsaturados de cadena larga, determinando un aumento de la peroxidación lo que, asociado a una menor ingesta de sus precursores en la dieta, sería uno de los factores involucrados en la génesis de la esteatosis observada en pacientes obesos ${ }^{27,28}$. También hemos observado un aumento en el contenido y la actividad del citocromo p450 2E1 (CYP2E1) en el hígado de estos pacientes. Esta enzima es un marcador de EO y su aumento se comelaciona positivamente con la severidad del daño hepático ${ }^{29}$. La expresión hepática de la CYPE1 se encuentra aumentada en diabetes tipo 2 , IR, obesidad central y ayuno ${ }^{30}$. La insulina ejerce una acción supresora de la regulación de la CYPE1 por lo tanto, la IR podnía estar ligada a la activación de este sistema enzimático y de esta forma, al daño de la EHGNA ${ }^{9}$.

Finalmente, probablemente existe un factor genético asociado lo que explicaría la tendencia familiar de presentar EHGNA ${ }^{6,31}$.

Otro hallazgo importante fue la correlación del aumento de las aminotransferasas con la inflamación lobulillar y fibrosis, lo que concuerda con lo observado por otros autores ${ }^{21}$. Es importante considerar que

\section{REFERENCIAS}

1. Beluentani S, Saccoccio G, Masutti F, Croce LS, Brandi $G$, SAsSO F ET AL. Prevalence of chronic liver disease in the general population of Northern Italy: the Dionysis study. Hepatology 1994; 20: 1442-9.

2. Ruhl CE, Everhart JE. Determinants of the association of overweight with elevated serum alanine aminotransferase activity in the United State. Gastroenterology 2003; 124: 71-9.

3. WanLESS IR, LeNTZ JS. Fatty liver hepatitis (steatohepatitis) and obesity: Autopsy study with analysis of risk factors. Hepatology 1990; 12: 1106-10.

4. ANDERSEN T, GuUUd C. Liver morphology in morbid obesity: a literature study. IntJ Obes 1984; 8: 97-106.

5. Foster KJ, Grifrith AH, Dewbury K, Price CP, Wright R. Liver disease in patients with diabetes mellitus. Postgrad Med J 1980; 56: 767-72.

6. Wilner IR, Waters B, Patil SR, Reuben A, Morew J, REEIY CA. Ninety patients with nonalcoholic steatohepatitis: insulin resistance, familial tendency, and severity of disease. Am J Gastroenterol 2001; 96: 2957-61.

7. Ponachik J, Mancila C, Contreras J, Csendes A, Smok G, la causa más frecuente de elevación persistente de aminotransferasas y estudio etiológico negativo es la EHGNA $^{32,33}$.

Aún no existe una terapia específica para la EHGNA, aunque algunos estudios han demostrado mejoría con dieta y reducción de peso, logrando la normalización de las aminotransferasas en más de $50 \%$ de los casos ${ }^{34}$. En este aspecto, es interesante mencionar que en un grupo de pacientes obesos con EHGNA sometidos a cirugía bariátrica, observamos una mejońa histológica significativa ${ }^{35}$. Desde el punto de vista farmacológico, se ha descrito que la metformina podnía revertir las alteraciones histológicas de la EHGNA $^{36,37}$. Están en desarrollo estudios clínicos que intentan precisar el efecto que pueda tener ésta u otros sensibilizadores a la acción de la insulina ${ }^{38}$.

Concluimos que los pacientes con EHGNA presentan alteraciones de la tolerancia a la glucosa y síndrome metabólico con elevada frecuencia, aun en ausencia de obesidad; por lo tanto en estos casos se debería buscar dirigidamente elementos de síndrome metabólico y realizar una prueba de tolerancia a la glucosa oral dentro de su estudio. Esto permitirá implementar, en forma precoz, las medidas terapéuticas que permitan prevenir las complicaciones metabólicas y evitar la progresión del daño hepático.

CAVADA G ET AL Obesity: risk factor for steatohepatitis and hepatic fibrosis. Rev Méd Chile 2002; 130: 731-6.

8. MarchesinI G, Brizi M, Morsew-Labate AM, Bianchi G, Bugianesi E, McCuLough AJ et al. Association of nonalcoholic fatty liver disease with insulin resistance. Am J Med 1999; 107: 450-5.

9. Marceau P, Biron S, Hould FS, Marceau S, Simard S, THung SN. Liver pathology and the metabolic syndrome $\mathrm{X}$ in severe obesity. J Clin Endocrinol Metab 1999; 84: 1513-7.

10. Dixon JB, Bhathal PS, O’Brien PE. Nonalcoholic fatty liver disease: Predictors of nonalcoholic steatohepatitis and liver fibrosis in the severely obese. Gastroenterology 2001; 121: 91-100.

11. Ryan MC, Wilson AM, Siavin J, Best JD, Jenkins AJ, Desmond PV. Associations between liver histology and severity of the metabolic syndrome in subjects with nonalcoholic fatty liver disease. Diabetes Care 2005; 28: 1222-4.

12. Marchesini G, Brizi M, Bianchi G, Tomassetti S, Bugianesi E, LenZi M ET AL. Nonalcoholic fatty liver disease. A feature of the metabolic syndrome. Diabetes 2001; 50: 1844-50. 
13. American Diabetes Association. Diagnosis and classification of diabetes mellitus. Diabetes Care 2004; 27: S5-S10.

14. International Diabetes Federation: The IDF consensus worldwide definition of the metabolic syndrome. Available from http://www.idf.org/webdata/ docs/Metabolic_syndrome_definition.pdf. Accessed 2 September 2005.

15. Knobier H, Schattner A, Zhornicki T, Malnick SDH, Keter D, SoKolovskaya N et aL. Fatty liver-an additional and treatable feature of the insulin resistance syndrome. QJM 1999; 92: 73-9.

16. García-Monzón C, Martín-Pérez E, Iacono OL, Fernández-Bermejo M, Majano PL, Apolinario A et AL. Characterization of pathogenic and prognostic factors of nonalcoholic steatohepatitis associated with obesity. J Hepatol 2000; 33: 716-24.

17. Marchesini G, Bugianesi E, Forlani G, Cerreul F, LenZI M, Manini R ET aL. Nonalcoholic fatty liver, steatohepatitis, and the metabolic syndrome. $\mathrm{He}$ patology 2003; 37: 917-23.

18. GuzZaloni G, Grugni G, Minocci A, Moro D, MorabiTо F. Liver steatosis in juvenile obesity: correlations with lipid profile, hepatic biochemical parameters and glycemic and insulinemic responses to an oral glucose tolerance test. Int J Obesity 2000; 24: 772-6.

19. Vannin $P$, Forlani G, Marchesini G, Ciavareita A, Zou M, PISI E. The euglicemic clamp technique in patients with liver cimhosis. Horm Metabol Res 1984; 16: 341-3.

20. Cortez-Pinto H, Camilo ME, Baptista A, De Oliveira AG, De Moura MC. Non-alcoholic fatty liver: another feature of the metabolic syndrome? Clin Nutr 1999; 18: 353-8.

21. Paradis V, Periemuter G, Bonvoust F, Dargere D, Parfatt B, Vidaud $M$ et al. High glucose and hyperinsulinemia stimulate a potential mechanism involved in progression to fibrosis in nonalcoholic steatohepatitis. Hepatology 2001; 34: 738-44.

22. СомРоRтI M. Lipid peroxidation and cellular damage in toxic liver injury. Lab Invest 1985; 53: 599-623.

23. Fong DG, Nehra V, LiNdor KD, Buchman AL. Metabolic and nutritional considerations in nonalcoholic fatty liver. Hepatology 2000; 32: 3-10.

24. Sanyal AJ, Campbell-Sargent C, Mirshahi F, Rzzzo WB, Contos MJ ET AL. Nonalcoholic steatohepatitis: Association of insulin resistance and mitochondrial abnormalities. Gastroenterology 2001; 120: 1183-92.

25. DaY CA, James O. Steatohepatitis: a tale of two hits? Gastroenterology 1998; 114: 842-5.

26. Videia LA, Rodrigo R, Orelana M, Fernández V, TAPIA G, ConTRERAS J ET aL. Oxidative stress-related parameters in the liver nonalcoholic fatty liver disease patients. Clin Sci 2004; 106: 261-8.

27. Videla LA, Rodrigo R, Araya J, Poniachik J. Oxidative stress and depletion of hepatic long-chain polyunsaturated fatty acids may contribute to nonalcoholic fatty liver disease. Free Radic Biol Med 2004; 37: 1499-507.

28. Araya J, Rodrigo R, Videita LA, Thielemann L, Oreijana M, Pettinew P ET AL. Increase in long-chain polyunsaturated fatty acid $n-6 / n-3$ ratio in relation to hepatic steatosis in patients with non-alcoholic fatty liver disease. Clin Sci 2004; 106: 635-43.

29. Oreliana M, Rodrigo R, Vareia N, Araya J, PoniaCHIK J, CSENDes A et AL. Relationship between in vivo chlorzoxazone hydroxilation, hepatic cytochrome P450 2E1 content and liver injury in obese non-alcoholic fatty liver disease patients. Hepatol Res 2005; 28: (en prensa).

30. Oremana M, Guajardo V. Cytochrome P450 activity and its alteration in different diseases. Rev Méd Chile 2004; 132: 85-94.

31. Struben VMD, Hespenheide EE, Caldwell S. Nonalcoholic steatohepatitis and cryptogenic cirrhosis within kindreds. Am J Med 2000; 108: 9-13.

32. Sorbi D, McGIL DB, Thistie JL, Therneau TM, Henry J, LNDOR KD. An assessment of the role of liver biopsies in asymptomatic patients with chronic liver test abnormalities. Am J Gastroenterol 2000; 95: 3206-10.

33. Caldwell SH, Oelsner DH, IeZzoni JC, Hespenheide EE, BatTLE EH, DRISCOL CJ. Cryptogenic cirrhosis: clinical characterization and risk factors for underlying disease. Hepatology 1999; 29: 664-9.

34. ERIKSSON S, ERIKSSON KF, BondesSON L. Nonalcoholic steatohepatitis in obesity: a reversible condition. Acta Med Scand 1986; 220: 83-8.

35. Poniachik J, Meiys A, Contreras J, Valera JM, Burgos A, Papaprieto K et al. Mejonía histológica del hígado graso no alcohólico (HGNA) con la reducción de peso en pacientes obesos sometidos a cirugía bariátrica. Gastr Latinoam 2004; 15: 288 (A).

36. Lin HZ, Yang SQ, Chuckaree C, KuhajDa F, RonNet G, DieHL AM. Metformin reverses fatty liver disease in obese, leptin-deficient mice. Nat Med 2000; 6: 998-1003.

37. Nair S, Diehl AM, Wiseman M, Farr GH Jr, Perrilo RP. Metformin in the treatment of non-alcoholic steatohepatitis: a pilot open label trial. Aliment Pharmacol Ther 2004; 20: 23-8.

38. Valera JM, Poniachik J, Araya V, Contreras J, CaAmaño E. Protocolo doble ciego randomizado de metformina en la terapia del hígado graso no alcohólico (HGNA): informe preliminar. Gastr Latinoam 2004; 15: 281 (A). 